\title{
THE SPACE OF RETRACTIONS OF THE 2-SPHERE AND THE ANNULUS
}

\author{
BY \\ NEAL R. WAGNER( $\left.{ }^{(}\right)$
}

\begin{abstract}
Given a manifold $M$, there is an embedding $\Lambda$ of $M$ into the space of retractions of $M$, taking each point to the retraction of $M$ to that point. Considering $\Lambda$ as a map into the connected component containing its image, we prove that $\Lambda$ is a weak homotopy equivalence for two choices of $M$, namely, the 2-sphere and the annulus.
\end{abstract}

Introduction. In [3], Borsuk introduced the study of spaces of retractions and proved a theorem, which, when specialized to 2-manifolds, says that the space of retractions of a closed disk is contractible (in itself).

Given a manifold $M$, there is a natural isometric embedding $\Lambda$ of $M$ into the space of retractions of $M$, defined by setting $\Lambda(x)$ equal to the retraction taking $M$ to $x$, for any $x \in M$. Let $\mathscr{L}(M)$ denote the component containing the image of $\Lambda$. Then $e v \circ \Lambda$ is the identity map on $M$, where $e v$ maps any retraction to the retraction evaluated at some basepoint of $M$. Hence the induced homomorphism $\Lambda_{*}: \pi_{n}(M) \rightarrow \pi_{n}(\mathscr{L}(M))$ is injective, for all $n$. In $\S 2$ and $\S 3$, we prove that $\Lambda_{*}$ is surjective for two choices of $M$, namely, the 2-sphere and the 2-dimensional annulus. Thus we prove that $\Lambda$ in these two cases is a weak homotopy equivalence. We also show that for this pair of 2-manifolds, $\mathscr{L}(M)$ is just the space of nondeformation retractions of $M$. Hence these results show that the space of nondeformation retractions of the 2-sphere (resp. the annulus) has the same homotopy groups as the 2-sphere (resp. the annulus).

The proofs rely heavily on a selection theorem of E. Michael, which is stated in $\S 1$. The proof in $\$ 3$ requires certain results from conformal mapping theory which are also stated in $\S 1$.

1. Preliminaries. Throughout this paper we use certain standard notation, namely, $E^{n}$ for Euclidean $n$-space, $S^{n}$ for the unit $n$-sphere in $E^{n+1}, B^{n}$ for the unit

Presented to the Society January 23, 1970; received by the editors March 23, 1970 and, in revised form, November 3, 1970.

AMS 1970 subject classifications. Primary 54C15, 54C35, 57A05; Secondary 54C65, 55D10.

Key words and phrases. Retract, retraction, two-manifold, two-sphere, annulus, homotopy equivalence, weak homotopy equivalence, function space, compact-open topology, selection.

( ${ }^{1}$ The material in this paper appeared in a different form as part of the author's doctoral dissertation, written under the direction of Professor M.-E. Hamstrom at the University of Illinois, Urbana. The author would like to thank Professor Hamstrom and the referee for many helpful comments. Among other things, the referee suggested the present emphasis on the map $\Lambda$. 
$n$-ball in $E^{n}$, and $I$ for the closed unit interval $[0,1]$. In addition, we use $C_{\alpha}$ to denote the circle in $E^{2}$ with center at the origin and radius $\alpha$, and $A^{2}$ to denote the closed annulus bounded by $C_{1}$ and $C_{2}$. The symbol $A(J, K)$ denotes the closed annular region bounded by simple closed curves $J$ and $K$, where $J$ lies in the bounded complementary domain of $K$. Thus $A^{2}=A\left(C_{1}, C_{2}\right)$.

A retraction of a space $X$ is an idempotent map $\varphi$ of $X$ to itself, and the space $\varphi(X)$, denoted im $(\varphi)$, is called a retract of $X$. All function spaces in this paper will be given the compact-open topology, and since we treat only compact metric spaces, this topology will be the same as that given by the sup-metric. In addition to the notation $\mathscr{L}(M)$ defined in the introduction, we use $\mathscr{R}(X), \mathscr{D}(X)$, and $\mathscr{N}(X)$, respectively, for the space of retractions, deformation retractions, and nondeformation retractions of $X$, respectively. A map $f: X \rightarrow Y$ is a weak homotopy equivalence if the induced maps $f_{*}: \pi_{n}\left(X, x_{0}\right) \rightarrow \pi_{n}\left(Y, f\left(x_{0}\right)\right)$ are isomorphisms for all $n$, where $x_{0}$ is some basepoint of $X$. The existence of a weak homotopy equivalence implies that the two spaces involved have the same homotopy and integral singular homology groups [16, p. 406].

To motivate later work, we now present a modified proof of a theorem of Borsuk [3, p. 197]. The proof is almost a direct translation of the proof by Alexander [1] that the space of homeomorphisms of an $n$-ball, keeping the boundary fixed, is contractible. Since we are only concerned with 2-manifolds, we give the theorem for the disk, even though it is true for much more general spaces.

\subsection{THEOREM (BORSUK). The space $\mathscr{R}\left(B^{2}\right)$ is contractible in itself.}

Proof. For each $t \in I$, define a retraction $\rho_{t}$ of $B^{2}$ by letting $\rho_{t}$ be the identity on the disk inside $C_{1-t}$, and letting it map $A\left(C_{1-t}, C_{1}\right)$ radially to $C_{1-t}$. Next, for $0 \leqq t<1$, define a homeomorphism $h_{t}: B^{2} \rightarrow \rho_{t}\left(B^{2}\right)$ by $h_{t}(u)=(1-t) u$, for $u \in B^{2}$. Finally, define a contraction $\Theta_{t}$ of $\mathscr{R}\left(B^{2}\right)$ by

$$
\Theta_{t}(\varphi)=h_{t} \circ \varphi \circ h_{t}^{-1} \circ \rho_{t}, \quad \text { for } 0 \leqq t<1,
$$

and $\Theta_{1}(\varphi)=\rho_{1}$. We see that $\Theta_{t}(\varphi)$ always belongs to $\mathscr{R}\left(B^{2}\right)$, since $h_{t} \circ \varphi \circ h_{t}^{-1}$ is an idempotent map whose image is contained in the image of $\rho_{t}$. Only continuity for $t=1$ needs verifying, and this presents no problems. Q.E.D.

As a generalization of the technique in the previous proof, we have the following:

1.2. Remark (Elbowroom Construction). Let $M$ be a manifold with boundary $\partial M$ and let $\partial M \times[0,2]$ be a collar of the boundary. For $t \in I$, let $\rho_{t}$ be the retraction of $M$ which is the identity outside $\partial M \times[0, t]$, and projects $\partial M \times[0, t]$ to $\partial M \times\{t\}$. Let $h_{t}: M \rightarrow \rho_{t}(M)$ be the homeomorphism given by the identity outside $\partial M \times[0,2]$ and by mapping $\partial M \times[0,2]$ linearly to $\partial M \times[t, 2]$. Then the homotopy $\Theta_{t}$ given by $\Theta_{t}(\varphi)=h_{t} \circ \varphi \circ h_{t}^{-1} \circ \rho_{t}$ provides a deformation of $\mathscr{R}(M)$ in itself such that the image of $\Theta_{1}$ consists of retractions of $M$ whose images do not meet $\partial M$.

For future reference, we now quote a selection theorem of E. Michael [14, p. 563]. The theorem is stated here in a weak form, as in [11, p. 528]. 
1.3. Theorem (Michael). Suppose that $B$ and $X$ are metric spaces, $B$ is (metrically) topologically complete, the (covering) dimension of $X$ does not exceed $n$, and that $Y$ is a closed subspace of $X$. Suppose, further, that $\tau$ is an open mapping of $B$ onto $X$ such that the collection of (point) inverses under $\tau$ is equi-LC $C^{n-1}$ (see Definition 1.4), and that we have a partial mapping e of $Y$ into $B$ such that, for $y \in Y$, $e(y) \in \tau^{-1}(y)$. Then there is a neighborhood $U$ of $Y$ such that e may be extended to a mapping $e^{*}$ of $U$ into $B$ such that, for $x \in U, e^{*}(x) \in \tau^{-1}(x)$. If each inverse under $\tau$ has vanishing homotopy groups of order $\leqq n-1$, then $U$ may be taken to be the entire space $X$.

1.4. Definition. In a metric space $B$, let $N(w, \varepsilon)$ denote the open neighborhood with center at $w \in B$ and radius $\varepsilon$. Recall that $B$ is $L C^{n}$ if for $w \in B$ and $\varepsilon>0$, there is a $\delta>0$ such that each mapping of an $m$-sphere $(m \leqq n)$ into $N(w, \delta)$ is nullhomotopic in $N(w, \varepsilon)$. Using the notation of Theorem 1.3, the collection of inverses under $\tau$ is equi- $L C^{n}$ if, for $x \in X, w \in \tau^{-1}(x)$, and $\varepsilon>0$, there is a $\delta>0$ such that, if $x^{\prime} \in X$, then every mapping of an $m$-sphere $(m \leqq n)$ into $\tau^{-1}\left(x^{\prime}\right) \cap N(w, \delta)$ is nullhomotopic in $\tau^{-1}\left(x^{\prime}\right) \cap N(w, \varepsilon)$.

We wish to state some results from conformal mapping theory which will be needed later. In the rest of this section, whenever the index $i$ occurs, we assume that it ranges over the set of positive integers. In what follows, we have omitted the word "respectively" a number of times.

Let $G$ and $G_{i}$ be open, connected, simply connected subsets of $E^{2}$ with boundaries the simple closed curves $J$ and $J_{i}$. Suppose these curves are images of similarly oriented homeomorphisms $f$ and $f_{i}$ with domain $C_{1}$. Let $p$ be a point of $G$ and $p_{i}$ a point of $G_{i}$. Then there are homeomorphisms $h$ and $h_{i}$ of $B^{2}$ onto $G \cup J$ and $G_{i} \cup J_{i}$ which are conformal on the interior of $B^{2}$ and uniquely determined by requiring that they map the origin to $p$ and $p_{i}$ and that their derivatives be real and positive at the origin [13, p. 70]. Moreover, if $\left\{p_{i}\right\}$ converges to $p$ and $\left\{f_{i}\right\}$ converges uniformly to $f$ on $C_{1}$, then $\left\{h_{i}\right\}$ converges uniformly to $h$ on $B^{2}$. (See [4], [5], [6, p. 191], and [9, p. 27]. We shall sometimes refer to this last fact as the "continuity property.") Finally, there are unique (not necessarily conformal) homeomorphisms $F$ and $F_{i}$ of $B^{2}$ onto $G \cup J$ and $G_{i} \cup J_{i}$ such that $F$ extends $f, F_{i}$ extends $f_{i}, F$ and $F_{i}$ map the origin to $p$ and $p_{i}$, and the sequence $\left\{F_{i}\right\}$ converges uniformly to $F$ on $B^{2}$.

Similar results hold for annular regions. Let $f, f_{i}, g$, and $g_{i}$ be similarly oriented homeomorphisms onto simple closed curves $J, J_{i}, K$, and $K_{i}$, where $f$ and $f_{i}$ have domain $C_{1}, g$ and $g_{i}$ have domain $C_{2}$, and $J$ and $J_{i}$ lie in the bounded complementary domains of $K$ and $K_{i}$. First, there is, for some $r>1$, a homeomorphism of the annulus $A\left(C_{1}, C_{r}\right)$ onto $A(J, K)$, which is conformal on the interior and is uniquely determined by the orientation of the boundary and the image of one boundary point $\left[6\right.$, p. 38]. Next, there are homeomorphisms $F$ and $F_{i}$ which map $A^{2}$ onto $A(J, K)$ and $A\left(J_{i}, K_{i}\right)$, which extend the maps $f, f_{i}, g$, and $g_{i}$, and which are uniquely determined by the angle change along the image of the closed line segment 
$I^{\prime}$ from $(1,0)$ to $(2,0)$. (This means the net angle change with respect to a point inside $J$. For a careful definition, see [11, p. 522].) Finally, we have the continuity property: if $\left\{f_{i}\right\}$ converges uniformly to $f$ on $C_{1},\left\{g_{i}\right\}$ converges uniformly to $g$ on $C_{2}$, and the angle change along the arcs $F_{i}\left(I^{\prime}\right)$ converges to the angle change along $F\left(I^{\prime}\right)$, then the sequence $\left\{F_{i}\right\}$ converges uniformly to $F$ on $A^{2}$. (See [6], [11], and $[15$, p. 45].)

2. The 2-sphere. In this section, the goal is to prove the following theorem.

2.1. THEOREM. The embedding $\Lambda: S^{2} \rightarrow \mathscr{L}\left(S^{2}\right)$ is a weak homotopy equivalence. In addition, $\mathscr{L}\left(S^{2}\right)=\mathscr{N}\left(S^{2}\right)$.

It will follow from work later in this section that $\mathscr{N}\left(S^{2}\right)$, the space of nondeformation retractions, is pathwise connected. It is then easy to see that $\mathscr{L}\left(S^{2}\right)=\mathscr{N}\left(S^{2}\right)$, the identity map being the only deformation retraction. As stated in the introduction, we need only show that the induced map

$$
\Lambda_{*}: \pi_{n}\left(S^{2}, u_{0}\right) \rightarrow \pi_{n}\left(\mathscr{N}\left(S^{2}\right), \Lambda\left(u_{0}\right)\right)
$$

is surjective, where $u_{0}$ is some basepoint of $S^{2}$.

Given a retraction $\varphi$ in $\mathscr{N}\left(S^{2}\right)$, we shall show in this section how to construct a canonical deformation in $\mathscr{N}\left(S^{2}\right)$ from $\varphi$ to $\Lambda(a(u))$, where $a$ is the antipodal map on $S^{2}$ and $u$ is a point of $S^{2} \mid \operatorname{im}(\varphi)$. If we could just continuously select, for each $\varphi \in \mathscr{N}\left(S^{2}\right)$, a point from $S^{2} \mid \operatorname{im}(\varphi)$, then we could easily show that the map $\Lambda$ above is a homotopy equivalence, with homotopy inverse the evaluation map taking $\varphi$ to $\varphi\left(u_{0}\right)$. In this section, we use Michael's Theorem to select such points from $S^{2} \backslash \operatorname{im}(\varphi)$, but a finite-dimensionality condition in the theorem limits our result to proving that $\Lambda$ is a weak homotopy equivalence.

In order to prove $\Lambda_{*}$ surjective, let $\Phi:\left(I^{n}, \partial I^{n}\right) \rightarrow\left(\mathscr{N}\left(S^{2}\right), \Lambda\left(u_{0}\right)\right)$ be a map, where $I^{n}$ is the unit $n$-cube and $\partial I^{n}$ is its boundary. For each $x \in I^{n}$, set $B(x)$ $=S^{2} \mid$ im $(\Phi(x))$, and let $B \subset I^{n} \times S^{2}$ be the set of all points $(x, u)$, where $x \in I^{n}$ and $u \in B(x)$.

\subsection{Lemma. The set $B$ defined above is an open subspace of $I^{n} \times S^{2}$.}

Proof. Let $\left(x_{0}, w_{0}\right) \in B$, i.e., let $w_{0} \in B\left(x_{0}\right)$. Choose a number $\eta>0$ so that $\operatorname{dist}\left(w_{0}, \operatorname{im}\left(\Phi\left(x_{0}\right)\right)\right) \geqq \eta$. Choose $\delta>0$ such that if $x \in I^{n}$ and $d\left(x, x_{0}\right)<\delta$, then $d\left(\Phi(x), \Phi\left(x_{0}\right)\right)<\eta / 2$. (Note that the first " $d$ " represents the usual distance in $E^{n}$, while the second " $d$ " is being used for the sup-metric on $\mathscr{N}\left(S^{2}\right)$.) Define an open neighborhood $U$ of $\left(x_{0}, w_{0}\right)$ in $I^{n} \times S^{2}$ by letting $U$ equal the set of all $(x, w)$ such that $d\left(x, x_{0}\right)<\delta$ and $d\left(w, w_{0}\right)<\eta / 2$. In order to show that $U \subset B$, we choose $(x, w) \in U$ and show that $(x, w) \in B$ or $w \in B(x)$. It will clearly suffice to show $\operatorname{dist}(w, \operatorname{im}(\Phi(x)))>0$. Let $q \in \operatorname{im}(\Phi(x))$ and note that $\Phi(x)(q)=q$. Thus we have

$$
d\left(w_{0}, q\right) \geqq d\left(w_{0}, \Phi\left(x_{0}\right)(q)\right)-d\left(\Phi\left(x_{0}\right)(q), \Phi(x)(q)\right)>\eta-(\eta / 2)=\eta / 2
$$


Hence

$$
d(w, q) \geqq d\left(w_{0}, q\right)-d\left(w_{0}, w\right)>\eta / 2-\eta / 2=0 .
$$

Now consider the projection $\tau_{1}$ of $I^{n} \times S^{2}$ onto $I^{n}$, and let $\tau$ be $\tau_{1}$ restricted to $B$, so that $\tau^{-1}(x)=\{x\} \times B(x)$.

2.3. LEMMA. The spaces and map defined above satisfy the hypotheses of Theorem 1.3 , i.e.,

(a) the space $B$ is topologically complete,

(b) the map $\tau$ is an open surjection,

(c) for each $x \in I^{n}$, the space $\tau^{-1}(x)$ has vanishing homotopy groups of order $\leqq n-1$, and

(d) the collection of inverses under $\tau$, i.e., the collection of sets $\{x\} \times B(x)$ for $x \in I^{n}$, is equi-LC $C^{n-1}$. (See Definition 1.4.)

Proof. Using Lemma 2.2, the fact that $\tau_{1}$ is open, and the fact that im $(\Phi(x))$ is an absolute retract, everything is clear except possibly (d). To prove (d), note first that equi- $L C^{n}$ is a topological property. Thus choose a metric on $I^{n} \times S^{2}$ which yields open neighborhoods of the form: an open "box" in $I^{n}$ crossed with an open disk in $S^{2}$. The equi- $L C^{n}$ property then becomes a triviality. Q.E.D.

Proof of Theorem 2.1. We are in a position to apply Theorem 1.3, with $X$ in the theorem equal to $I^{n}, Y$ equal to $\partial I^{n}, B$ and $\tau$ of the theorem as above, and $e: \partial I^{n} \rightarrow B$ defined by $e(y)=\left(y, a\left(u_{0}\right)\right)$, for each $y \in \partial I^{n}$, where $a$ is the antipodal map on $S^{2}$ and $u_{0}$ is the basepoint of $S^{2}$. Note that $\left(y, a\left(u_{0}\right)\right) \in B$ because $\Phi$ maps $\partial I^{n}$ to $\Lambda\left(u_{0}\right)$. Theorem 1.3 gives an extension $e^{*}$ of $e$, mapping $I^{n}$ into $B$. If $\tau_{2}$ is the projection of $I^{n} \times S^{2}$ onto $S^{2}$, then the map $\tau_{2} \circ e^{*}: I^{n} \rightarrow S^{2}$ satisfies $\tau_{2} \circ e^{*}(x)$ $\in B(x)$. Thus, corresponding to each $x \in I^{n}, \tau_{2} \circ e^{*}$ selects a point from the complement of im $(\Phi(x))$.

We now construct a (basepoint-preserving) homotopy in $\mathscr{N}\left(S^{2}\right)$ from the map $\Phi$ to the map $\Lambda \circ a \circ \tau_{2} \circ e^{*}$. Since the latter map is a representative of the image of $a \circ \tau_{2} \circ e^{*}$ under $\Lambda_{*}$, this will show that $\Lambda_{*}$ is surjective. A similar construction can be used to show that $\mathscr{N}\left(S^{2}\right)$ is pathwise connected.

For notational convenience, let $x^{\prime}$ stand for the point $\tau_{2} \circ e^{*}(x)$ in $S^{2} \mid \operatorname{im}(\Phi(x))$, where $x \in I^{n}$. A compactness argument shows that there is a number $\beta>0$ such that the great circle distance between $x^{\prime}$ and $\operatorname{im}(\Phi(x))$ is greater than $\beta$ for all $x \in I^{n}$.

In $S^{2}$, let $C(x)$ stand for the circle with center $x^{\prime}$ and radius $\beta$ (great circle distance). The circle $C(x)$, along with the component of $S^{2} \mid C(x)$ containing $x^{\prime}$, is disjoint from $\operatorname{im}(\Phi(x))$. Define a collection of homeomorphisms $h_{t}(x)$ of $S^{2}$ onto itself, for each $0 \leqq t<1$ and each $x \in I^{n}$. Let $h_{t}(x)$ take $C(x)$ to the circle with center $x^{\prime}$ and radius $(1-t) \beta+t \pi$, let $h_{t}(x)$ fix $x^{\prime}$ and its antipode $a\left(x^{\prime}\right)$, and extend $h_{t}(x)$ linearly along great circles through $x^{\prime}$. The map $h_{0}(x)$ is the identity on $S^{2}$, and the effect of $h_{t}(x)$, as $t$ tends to 1 , is to take the component of $S^{2} \backslash C(x)$ not containing $x^{\prime}$ to the point $a\left(x^{\prime}\right)$. It is clear that these homeomorphisms are jointly continuous in the sup-metric in both variables $t$ and $x$. 
We can now define a homotopy $\Theta_{t}$ in $\mathscr{N}\left(S^{2}\right)$ by setting, for each $x \in I^{n}$,

$$
\Theta_{t}(x)=h_{t}(x) \circ \Phi(x) \circ h_{t}(x)^{-1}, \text { for } 0 \leqq t<1,
$$

and setting $\Theta_{1}(x)=\Lambda\left(a\left(x^{\prime}\right)\right)=\Lambda \circ a \circ \tau_{2} \circ e^{*}(x)$. Here $\Theta_{0}(x)=\Phi(x)$, and $\Theta_{t}(y)$ $=\Lambda\left(u_{0}\right)$, for $y \in \partial I^{n}$ and $t \in I$. Also $\Theta_{t}(x) \in \mathscr{N}\left(S^{2}\right)$ for any $t$ and $x$, and $\Theta_{t}$ is clearly continuous when $t \neq 1$.

The continuity of $\Theta_{t}$ when $t=1$ follows from the special nature of the construction. If $D(x)$ denotes the component of $S^{2} \backslash C(x)$ containing $a\left(x^{\prime}\right)$, then im $(\Phi(x))$ is contained in $D(x)$. Thus im $\left(\Theta_{t}(x)\right)$ is contained in $h_{t}(x)(D(x))$, and the latter set is an open disk centered at $a\left(x^{\prime}\right)$ whose radius we can make as small as we like by taking $t$ sufficiently close to 1 . Q.E.D.

REMARK. The methods in this section cannot be used for $S^{m}(m>2)$ because the complement of a retract of $S^{m}$ need not have vanishing homotopy groups. In fact, the solid Alexander Horned Sphere is an absolute retract, and so is a retract of $S^{3}$, but its complement is not simply connected.

3. The annulus. Recall that the annulus $A^{2}$ is the set $A\left(C_{1}, C_{2}\right)$ consisting of points $u \in E^{2}$ such that $1 \leqq|u| \leqq 2$. In this section we use methods very similar to those in $\$ 2$ to obtain the following result.

3.1. THEOREM. The embedding $\Lambda: A^{2} \rightarrow \mathscr{L}\left(A^{2}\right)$ is a weak homotopy equivalence. In addition, $\mathscr{L}\left(A^{2}\right)=\mathscr{N}\left(A^{2}\right)$.

If $\varphi$ is a retraction of $A^{2}$, then $\varphi^{2}=\varphi$, so that the degree of $\varphi$ is either 1 or 0 , and hence $\varphi$ is either homotopic to the identity or to a constant map. The first Čech cohomology group of the image of $\varphi$ is thus determined, depending on whether $\varphi$ is a deformation retraction or not, and correspondingly the image of $\varphi$ separates $E^{2}$ into two components, or its complement is connected. In any compact manifold, there is a number $\varepsilon$ such that any two self maps whose distance apart is less than $\varepsilon$ are homotopic. Thus $\mathscr{N}\left(A^{2}\right)$ and $\mathscr{D}\left(A^{2}\right)$ are at least a distance $\varepsilon$ apart. (In fact, it is not hard to show that they are a distance 2 apart.) Finally, it will follow from work later in this section that $\mathscr{N}\left(A^{2}\right)$ is pathwise connected, so that $\mathscr{L}\left(A^{2}\right)=\mathscr{N}\left(A^{2}\right)$.

Using Remark 1.2 and some conformal mapping theory, one can also prove that $\mathscr{D}\left(A^{2}\right)$ is pathwise connected. A detailed study of the space of deformation retractions of any compact 2-manifold will appear elsewhere.

As in $\S 2$, we can finish the proof of Theorem 3.1 by showing that the induced map $\Lambda_{*}$ is surjective. The idea of the proof is similar to that of Theorem 2.1 , but here most of the work will involve verifying that the hypotheses of Theorem 1.3 are satisfied.

Let $\Phi:\left(I^{n}, \partial I^{n}\right) \rightarrow\left(\mathscr{N}\left(A^{2}\right), \Lambda(3 / 2,0)\right)$ be a map, where $(3 / 2,0)$ is the basepoint of $A^{2}$. Using the elbowroom construction of Remark 1.2, we can assume that im $(\Phi)$ consists of retractions whose images do not meet $\partial A^{2}$. It turns out that the crucial problem in this section is to produce continuously, for each $x \in I^{n}$, an arc 
in $A^{2} \mid \operatorname{im}(\Phi(x))$ from $C_{1}$ to $C_{2}$, lying except for its endpoints in the interior of $A^{2}$. We can then produce a homotopy from $\Phi$ to a map in the image of $\Lambda_{*}$, proving that $\Lambda_{*}$ is surjective.

For any $\varphi \in \mathscr{N}\left(A^{2}\right)$, denote $A^{2} \mid \operatorname{im}(\varphi)$ by $M(\varphi)$. Let $\mathscr{H}\left(I, A^{2}\right)$ stand for the space of homeomorphisms $h: I \rightarrow A^{2}$ such that $h$ takes 0 to $C_{1}, 1$ to $C_{2}$, and the interior of $I$ to the interior of $A^{2}$. Similarly, let $\mathscr{H}(I, M(\varphi))$ be the space of homeomorphisms $h: I \rightarrow M(\varphi)$ satisfying the other three conditions. Define $\mathscr{B}$ to be the subset of $I^{n} \times \mathscr{H}\left(I, A^{2}\right)$ consisting of points $(x, h)$ such that $x \in I^{n}$ and $h \in \mathscr{H}(I, M(\Phi(x)))$. Finally, let $\tau_{1}$ be the projection of $I^{n} \times \mathscr{H}\left(I, A^{2}\right)$ onto $I^{n}$, and let $\tau$ be the restriction of $\tau_{1}$ to $\mathscr{B}$, so that $\tau^{-1}(x)=\{x\} \times \mathscr{H}(I, M(\Phi(x)))$.

3.2. THEOREM. The spaces and map defined above satisfy the hypotheses of Theorem 1.3, i.e.,

(a) the space $\mathscr{B}$ is topologically complete,

(b) the map $\tau$ is an open surjection,

(c) for each $x \in I^{n}$, the space $\tau^{-1}(x)$ has vanishing homotopy groups of order $\leqq n-1$, and

(d) the collection of inverses under $\tau$ is equi- $L C^{n-1}$.

The theorem will follow from the next two lemmas without much difficulty.

3.3. Lemma. For each $\varphi \in \mathscr{N}\left(A^{2}\right)$, the space $\mathscr{H}(I, M(\varphi))$ is contractible in itself.

Proof. Unless the contrary is explicitly stated, coordinates of all points will be rectangular.

Since im $(\varphi)$ is contractible, for $\varphi \in \mathscr{N}\left(A^{2}\right)$, it is easy to see that $M(\varphi)$ is homeomorphic to $A^{2}$ minus the closed disk with radius $1 / 4$ and center $(-3 / 2,0)$. Denote this set by $Z$. Let $\mathscr{H}(I, Z)$ be the subspace of $\mathscr{H}\left(I, A^{2}\right)$ consisting of those homeomorphisms which map into $Z$. We shall show that $\mathscr{H}(I, Z)$ is contractible.

Let $A^{\prime}=\left\{\left(u_{1}, u_{2}\right) \in E^{2}: 1 \leqq u_{1} \leqq 2\right\}$ be the universal covering space of $A^{2}$, where the covering projection $p$ is defined by $p\left(u_{1}, u_{2}\right)=\left(u_{1} \cos u_{2}, u_{1} \sin u_{2}\right)$. For any $h \in \mathscr{H}(I, Z)$, the interior of $A^{2}$ minus the image of $h$ is an open disk in $A^{2}$. Since it is simply connected, it is covered in $A^{\prime}$ by disjoint copies which project homeomorphically into $A^{2}$. In $A^{\prime}$ choose the copy of this open disk which contains the closed disk with radius $1 / 4$ and center $(3 / 2, \pi)$. Lift $h$ to maps $h^{\prime}$ and $h^{\prime \prime}$ of $I$ into $A^{\prime}$ whose images lie on the boundary of the chosen open disk, such that the second coordinate of $h^{\prime}(t)$ is $2 \pi$ smaller than the second coordinate of $h^{\prime \prime}(t)$, for $t \in I$. (Please refer to the Figure.) Let $\alpha_{h}$ be the second coordinate of $h^{\prime}(0)$ and let $\beta_{h}$ be the second coordinate of $h^{\prime}(1)$.

We now define, for each $h \in \mathscr{H}(I, Z)$, an isotopy $H_{t}(h)$ of $A^{\prime}$. The first coordinates of all points are left fixed, and $H_{t}(h)$ fixes points of $A^{\prime}$ with first coordinate $u_{1}$ between $9 / 8$ and $15 / 8$. Points with first coordinate $u_{1}=1$ have their second coordinate decreased by $t \alpha_{h}$, and for $u_{1}$ between 1 and $9 / 8$, the amount of decrease tends linearly to zero when $u_{1}=9 / 8$. Similarly, points with $u_{1}=2$ have their second 

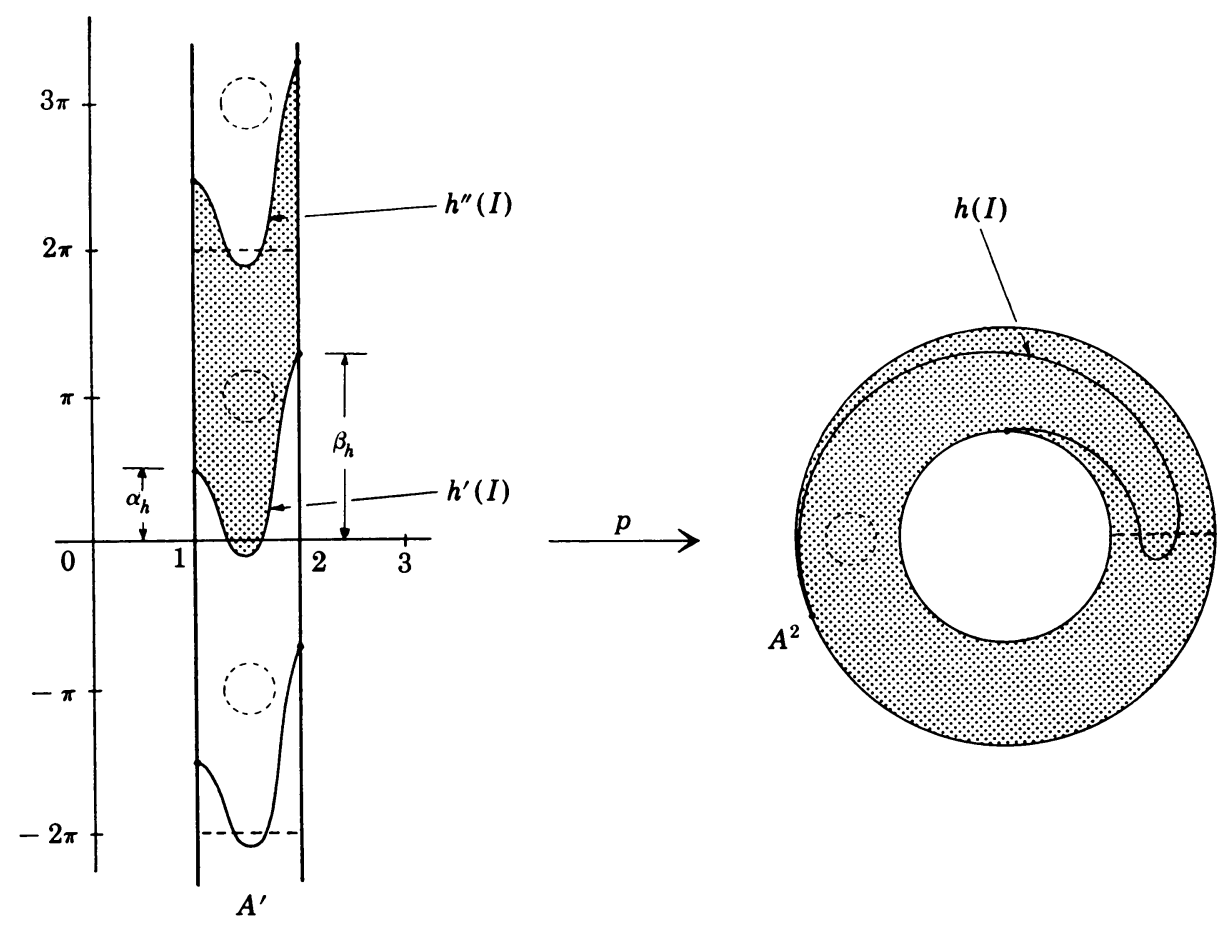

coordinate decreased by $t \beta_{h}$, and for $u_{1}$. between $15 / 8$ and 2 , the amount of decrease tends linearly to zero at $u_{1}=15 / 8$. The effect of $H_{t}(h)$, as $t$ goes from 0 to 1 , is to continuously shift $h^{\prime}(0)$ and $h^{\prime}(1)$ to the points $(1,0)$ and $(2,0)$, respectively. Let $\mathscr{H}^{\prime}(I, Z)$ be the subspace of $\mathscr{H}(I, Z)$ consisting of maps $h$ such that $h^{\prime}(0)=(1,0)$ and $h^{\prime}(1)=(2,0)$. (Notice that $\mathscr{H}^{\prime}$ is defined using the special "lifted" map $h^{\prime}$.) There are various ways of seeing that any $h \in \mathscr{H}^{\prime}(I, Z)$ is homotopic, keeping the endpoints fixed, to the map $h_{0}$, defined by $h_{0}(t)=(1+t, 0)$, for $t \in I$. It then follows from [8, p. 89] that $h$ is isotopic to $h_{0}$, keeping the endpoints fixed. Clearly, $p \circ H_{t}(h) \circ p^{-1}$ is always a homeomorphism of $A^{2}$ onto itself, and the homotopy $K_{t}$, defined by $K_{t}(h)=p \circ H_{t}(h) \circ p^{-1} \circ h$, gives a strong deformation retraction of $\mathscr{H}(I, Z)$ onto $\mathscr{H}^{\prime}(I, Z)$.

Let $D_{2}$ denote the closure of $Z$, which is just $A^{2}$ minus the open disk with radius $1 / 4$ and center $(-3 / 2,0)$. Let $\mathscr{H}_{0}\left(D_{2}\right)$ be the identity component of the space of homeomorphisms of $D_{2}$ onto itself, keeping the boundary curves pointwise fixed. For each $h \in \mathscr{H}^{\prime}(I, Z)$, we are going to define a canonical homeomorphism $k(h) \in \mathscr{H}_{0}\left(D_{2}\right)$, where "canonical" means that $k(h)$ is uniquely determined by $h$ and varies continuously with $h$. We also want $k(h)$ to map $(1+t, 0)$ to $h(t)$, for $t \in I$.

First, we define a homeomorphism $k^{\prime}(h)$ of $D_{2}^{\prime}$ onto itself, where $D_{2}^{\prime}$ is $A^{\prime}$ minus the collection of open disks above the open disk with radius $1 / 4$ and center $(-3 / 2,0)$. The space $D_{2}^{\prime}$ is a covering space of $D_{2}$, and we use the same symbol $p$ 
for the restriction of the previous covering projection. We shall define $k^{\prime}(h)$ on the closed annular region consisting of points $\left(u_{1}, u_{2}\right)$ with $u_{1}$ between 1 and $2, u_{2}$ between 0 and $2 \pi$, and the distance from $\left(u_{1}, u_{2}\right)$ to $(3 / 2, \pi) \geqq 1 / 4$. We then use translates of this definition on the rest of $D_{2}^{\prime}$. The homeomorphism $k^{\prime}(h)$ will be uniquely determined by the following four boundary conditions:

(1) $k^{\prime}(h)$ is the identity on the line segments from $(1,0)$ to $(1,2 \pi)$ and from $(2,0)$ to $(2,2 \pi)$,

(2) $k^{\prime}(h)(1+t, 0)=h^{\prime}(t)$ and $k^{\prime}(h)(1+t, 2 \pi)=h^{\prime \prime}(t)$, for $t \in I$,

(3) $k^{\prime}(h)$ is the identity on the circle with radius $1 / 4$ and center $(3 / 2, \pi)$, and

(4) the angle change is zero, as determined by the image of the segment from $(1, \pi)$ to $(5 / 4, \pi)$ and by using the points $(3 / 2, \pi)$ as origin for polar coordinates.

We define $k(h)$ to be the homeomorphism $p \circ k^{\prime}(h) \circ p^{-1}$. In $\S 1$, we stated that $k^{\prime}(h)$ has the continuity property $\left(k^{\prime}(h)\right.$ varies continuously with $\left.h\right)$, and it is clear that $k(h)$ also has this continuity property.

Since any map $h \in \mathscr{H}^{\prime}(I, Z)$ is isotopic, keeping the endpoints fixed, to $h_{0}$, where $h_{0}(t)=(1+t, 0)$, for $t \in I$, we see that $k$ will induce a path of homeomorphisms of $D_{2}$, keeping the boundary curves fixed, from $k(h)$ to the identity on $D_{2}$. Hence $k(h)$ belongs to $\mathscr{H}_{0}\left(D_{2}\right)$.

It was proved in [15] that $\mathscr{H}_{0}\left(D_{2}\right)$ is contractible in itself. (For Theorem 3.2(c) we only need the result from [10] that $\mathscr{H}_{0}\left(D_{2}\right)$ has vanishing homotopy groups.) Using the map $k$, the contraction of $\mathscr{H}_{0}\left(D_{2}\right)$ induces a contraction of $\mathscr{H}^{\prime}(I, Z)$, which is a deformation retract of $\mathscr{H}(I, Z)$. Hence the latter space is contractible. Q.E.D.

Next we prove a result which is perhaps known in some general formulation to experts, but has apparently not appeared in print.

\subsection{Lemma. The space $\mathscr{H}\left(I, A^{2}\right)$ is locally contractible.}

Proof. Let $h_{1}$ be any map in $\mathscr{H}\left(I, A^{2}\right)$, and let $U$ be the compact-open neighborhood of $h_{1}$ consisting of maps $h$ whose distance in the sup-metric from $h_{1}$ is less than $\varepsilon$, for some $\varepsilon>0$. Corresponding to each $h \in U$, we shall produce a homeomorphism $k(h) \in \mathscr{H}\left(A^{2}\right)$, the space of homeomorphisms of $A^{2}$ onto itself, not necessarily fixing the boundary. For any $h \in U, k(h)$ will be uniquely determined by the following four conditions:

(1) $k(h)$ is a rotation on $C_{1}$ so that $k(h)\left(h_{1}(0)\right)=h(0)$,

(2) $k(h)$ is a rotation on $C_{2}$ so that $k(h)\left(h_{1}(1)\right)=h(1)$,

(3) $k(h)(u)=h \circ h_{1}^{-1}(u)$, for $u \in h_{1}(I)$, and

(4) $k(h)\left(u_{0}\right)=u_{0}$, for some fixed $u_{0}$ in the interior of $A^{2}$ which misses all the sets $h(I)$, for $h \in U$.

We can construct $k(h)$ in a manner similar to the construction of the previous $k(h)$ in Lemma 3.3. As before, $k(h)$ will vary continuously with $h$, and $k\left(h_{1}\right)$ is the identity map on $A^{2}$. Also $k(h) \circ h_{1}=h$. 
Set $U^{\prime}$ equal to the set of all $h^{\prime} \in \mathscr{H}\left(A^{2}\right)$ within a distance $\varepsilon$ of the identity map on $A^{2}$, where $\varepsilon$ is the same number used to define $U$. The space $\mathscr{H}\left(A^{2}\right)$ is locally contractible [11, p. 524], so let $W^{\prime}$ be a neighborhood of the identity which is contractible in $U^{\prime}$. Let $W$ be a neighborhood of $h_{1}$ in $U$ such that $k(W) \subset W^{\prime}$, and let $W^{\prime} \circ h_{1}$ be the set of all $h \in \mathscr{H}\left(I, A^{2}\right)$ such that $h=h^{\prime} \circ h_{1}$, for some $h^{\prime} \in W^{\prime}$. It is easy to see that $W \subset W^{\prime} \circ h_{1} \subset U^{\prime} \circ h_{1} \subset U$, and thus $W^{\prime} \circ h_{1}$ is a neighborhood of $h_{1}$ in $U$ which is contractible in $U$. Q.E.D.

Proof of Theorem 3.2. Using a technique from [7, p. 107], let us first show that $\mathscr{H}\left(I, A^{2}\right)$ is topologically complete. The space $\mathscr{C}\left(I, A^{2}\right)$ of all continuous functions of $I$ into $A^{2}$ is complete in the sup-metric, and it has as a closed subspace the space $\mathscr{C}_{0}\left(I, A^{2}\right)$ of all maps taking 0 into $C_{1}$ and 1 into $C_{2}$. For each positive integer $n$, consider the subspace of $\mathscr{C}_{0}\left(I, A^{2}\right)$ consisting of all functions $g$ such that $\sup \left\{\operatorname{diam}\left(g^{-1}(u)\right): u \in g(I)\right\}$, sup $\left\{\alpha: g(\alpha) \in C_{1}\right\}$, and $1-\inf \left\{\alpha: g(\alpha) \in C_{2}\right\}$ are all less than $1 / n$. These subspaces are open in $\mathscr{C}_{0}\left(I, A^{2}\right)$ and their intersection is $\mathscr{H}\left(I, A^{2}\right)$, so that the latter, as a $G_{\delta}$ in $\mathscr{C}_{0}\left(I, A^{2}\right)$, is topologically complete.

A proof very similar to that of Lemma 2.2 will show that $\mathscr{B}$ is open in $I^{n} \times \mathscr{H}\left(I, A^{2}\right)$, and hence is topologically complete. The rest of the theorem now follows easily from Lemmas 3.3 and 3.4 as in the proof of Lemma 2.3. Q.E.D.

Proof of Theorem 3.1. Please refer to the statement of Theorem 3.2 and the discussion immediately preceding it. Let $g_{0}: I \rightarrow A^{2}$ be the map defined by $g_{0}(t)$ $=(-1-t, 0)$, for $t \in I$. Since $\Phi(y)=\Lambda(3 / 2,0)$, for $y \in \partial I^{n}$, we see that $g_{0}(I) \subset M(\Phi(y))$ or $\left(y, g_{0}\right) \in \mathscr{B}$, when $y \in \partial I^{n}$. Let $e: \partial I^{n} \rightarrow \mathscr{B}$ be the map $e(y)$ $=\left(y, g_{0}\right)$. Theorem 1.3 gives an extension $e^{*}: I^{n} \rightarrow \mathscr{B}$. We have arranged things so that, for any $x \in I^{n}, \tau_{2} \circ e^{*}(x)$ is an element of $\mathscr{H}(I, M(\Phi(x)))$, where $\tau_{2}$ is the projection of $I^{n} \times \mathscr{H}\left(I, A^{2}\right)$ onto $\mathscr{H}\left(I, A^{2}\right)$. Thus, $\tau_{2} \circ e^{*}(x)$ gives the desired canonical arc from $C_{1}$ to $C_{2}$ in $A^{2} \mid \operatorname{im}(\Phi(x))$. Notice also that $\Phi(x)(3 / 2,0)$ gives a canonical point in the interior of $A^{2}$ which misses the image of $\tau_{2} \circ e^{*}(x)$.

By the same construction that was used in the proofs of Lemmas 3.3 and 3.4 (using the universal covering space of $A^{2}$ ), we get a canonical homeomorphism $k(x)$ of $A^{2}$ onto itself, for each $x \in I^{n}$. The homeomorphism $k(x)$ will be uniquely determined by the following four conditions:

(1) $k(x)$ is a rotation on $C_{1}$ taking $\tau_{2} \circ e^{*}(x)(0)$ to $(-1,0)$,

(2) $k(x)$ is a rotation on $C_{2}$ taking $\tau_{2} \circ e^{*}(x)(1)$ to $(-2,0)$,

(3) $k(x) \circ\left(\tau_{2} \circ e^{*}(x)\right)=g_{0}$, and

(4) $k(x)(\Phi(x)(3 / 2,0))=(3 / 2,0)$.

The above construction yields a map $\Phi^{\prime}:\left(I^{n}, \partial I^{n}\right) \rightarrow\left(\mathscr{N}\left(A^{2}\right), \Lambda(3 / 2,0)\right)$, defined by $\Phi^{\prime}(x)=k(x) \circ \Phi(x) \circ k(x)^{-1}$, whose image consists of retractions with images in the complement of the segment from $(-1,0)$ to $(-2,0)$. We now cut $A^{2}$ along this segment to yield a closed disk with a collection of retractions, and then use Theorem 1.1 to see that these are homotopic to the constant retraction to $(3 / 2,0)$. From the proof of Theorem 1.1, it is clear that the retractions of this disk will yield retractions of $A^{2}$, after identifying the cut portion. Thus $\Phi^{\prime}$ is homotopic to a 
constant map with image $\Lambda(3 / 2,0)$. This defines a homotopy of $\Phi$ to a map $\Psi$ such that

$$
\begin{aligned}
\Psi(x) & =k(x)^{-1} \circ \Lambda(3 / 2,0) \circ k(x) \\
& =k(x)^{-1} \circ \Lambda(3 / 2,0)=\Lambda\left(k(x)^{-1}(3 / 2,0)\right) .
\end{aligned}
$$

Hence $\Psi^{\prime}(x)$ lies in the image of $\Lambda$, so that $\Lambda_{*}$ is surjective. Q.E.D.

\section{REFERENCES}

1. J. W. Alexander, On the deformation of an n-cell, Proc. Nat. Acad. Sci. U.S.A. 9 (1923), 406-407.

2. K. Borsuk, Theory of retracts, Monografie Mat., Tom 44, PWN, Warsaw, 1967. MR 35 \#7306.

3. - Concerning the set of retractions, Colloq. Math. 18 (1967), 197-201. MR 36 \#2126.

4. R. Courant, Über eine Eigenschaft der Abbildungsfunctionen bei konformer Abbildung, Gött. Nachr. (1914), 101-109.

5. —_ Bemerkung zu meiner Note: "Über eine Eigenschaft...", Gött. Nachr. (1922), 69-70.

6. — Dirichlet's principle, conformal mapping, and minimal surfaces, Interscience, New York, 1950. MR 12, 90.

7. E. Dyer and M.-E. Hamstrom, Completely regular mappings, Fund. Math. 45 (1958), 103-118. MR 19, 1187.

8. D. B. A. Epstein, Curves on 2-manifolds and isotopies, Acta Math. 115 (1966), 83-107. MR 35 \#4938.

9. C. Gattegno and A. Ostrowski, Représentation conforme a la frontière; domaines généraux, Mém. Sci. Math., no. 109, Gauthier-Villars, Paris, 1949. MR 11, 425.

10. M.-E. Hamstrom, Some global properties of the space of homeomorphisms on a disc with holes, Duke Math. J. 29 (1962), 657-662. MR 26 \#745.

11. M.-E. Hamstrom and E. Dyer, Regular mappings and the space of homeomorphisms on a 2-manifold, Duke Math. J. 25 (1958), 521-531. MR 20 \#2695.

12. S.-T. Hu, Homotopy theory, Pure and Appl. Math., vol. 8, Academic Press, New York, 1959. MR 21 \#5186.

13. A. I. Markuševič, Theory of functions of a complex variable. Vol. III, GITTL, Moscow, 1950; English transl., Prentice-Hall, Englewood Cliffs, N. J., 1967. MR 12, 87; MR 35 \#6799.

14. E. Michael, Continuous selections. II, Ann. of Math. (2) 64 (1956), 562-580. MR 18, 325.

15. H. R. Morton, The space of homeomorphisms of a disc with $n$ holes, Illinois J. Math. 11 (1967), 40-48. MR $34 \# 5066$.

16. E. H. Spanier, Algebraic topology, McGraw-Hill, New York, 1966. MR 35 \#1007.

UNIVERSITY OF TEXAS,

El Paso, Texas 79999 